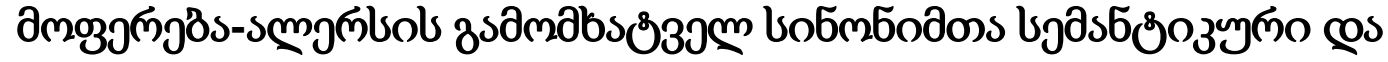

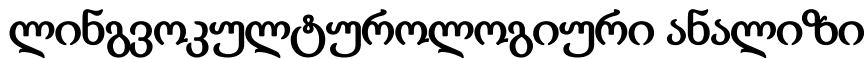

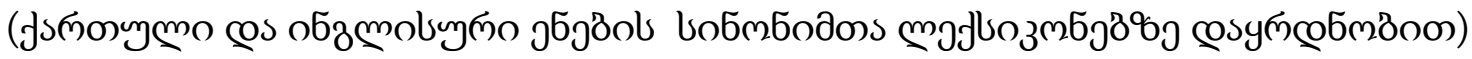

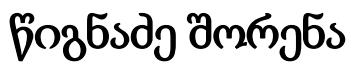

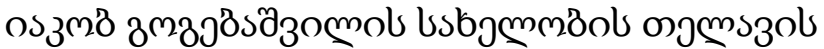

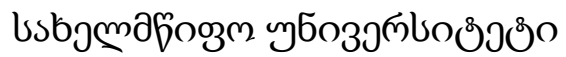

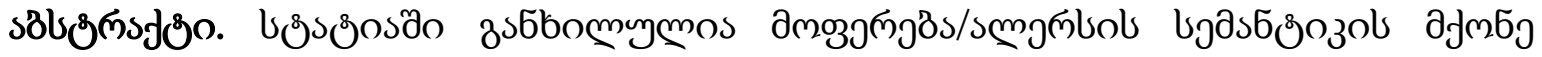

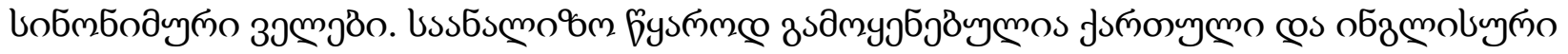

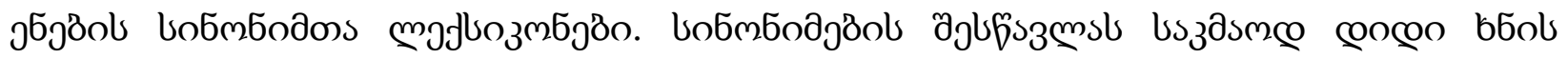

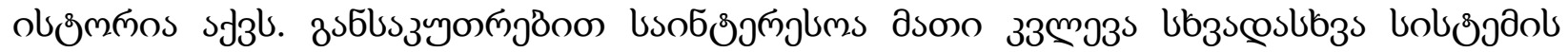

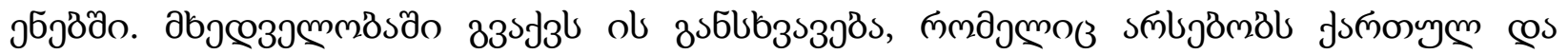

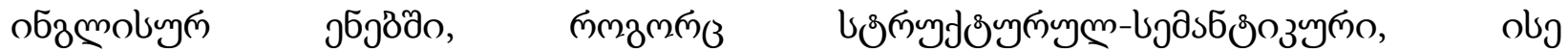

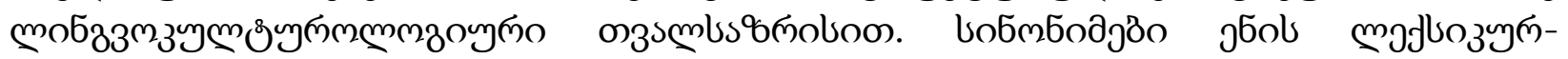

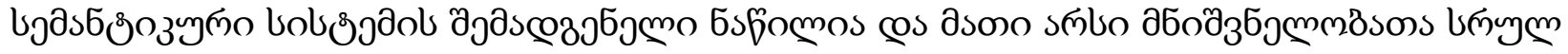

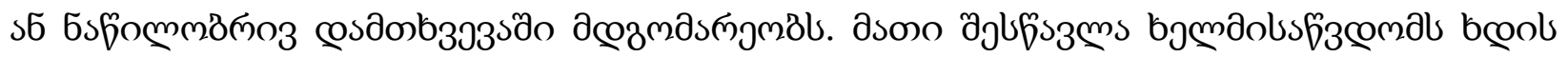

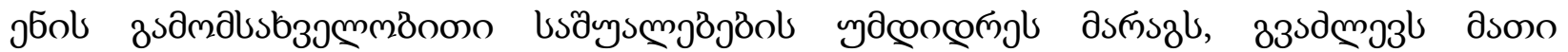

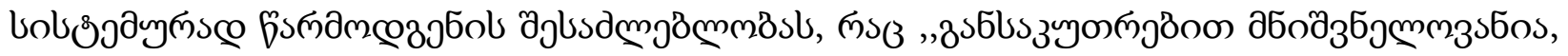

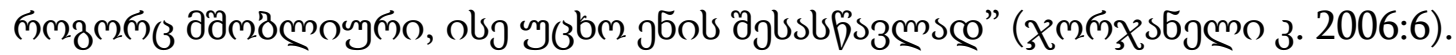

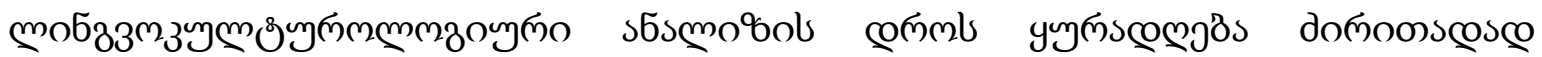

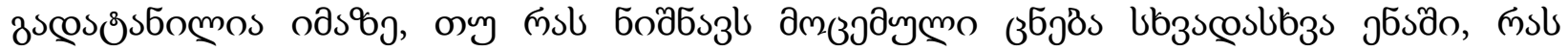

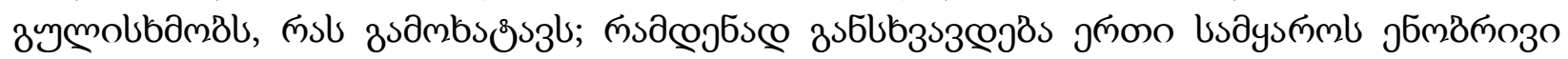

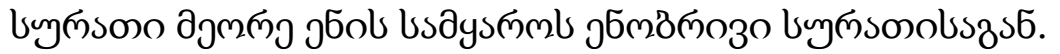

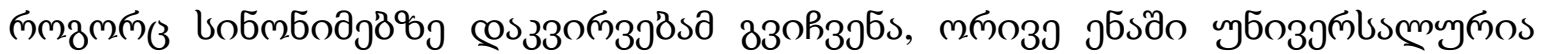

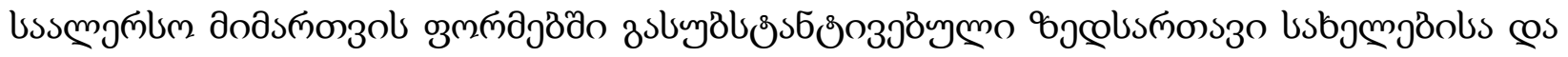

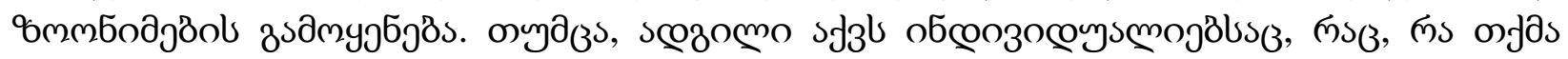

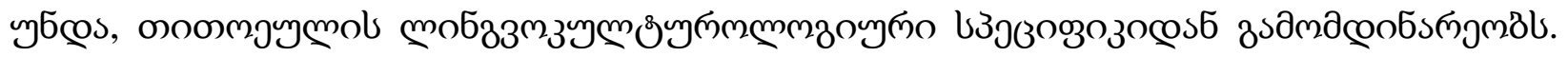

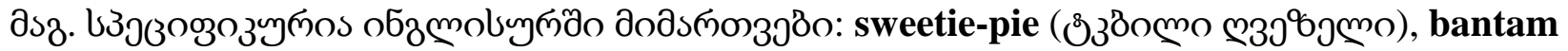

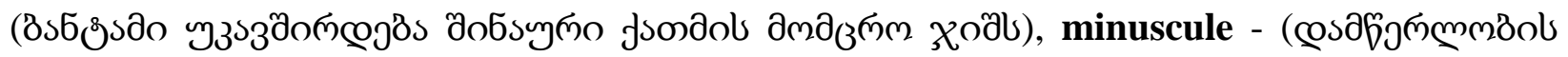

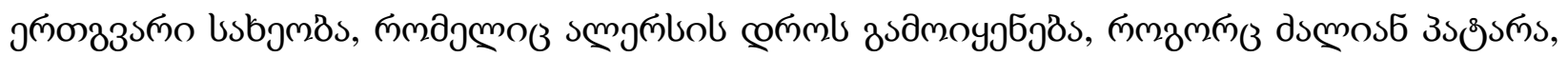

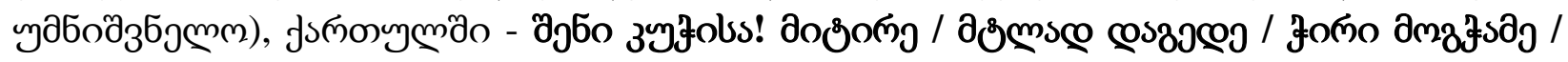

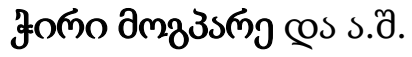

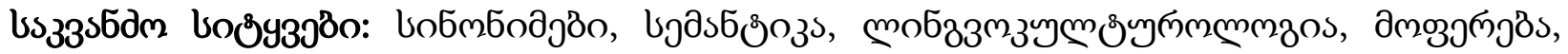


sলmgn̆lo.

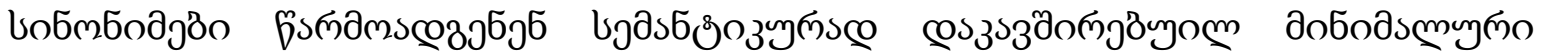

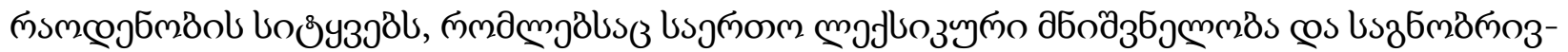

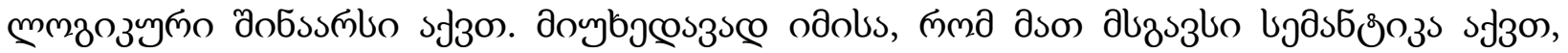

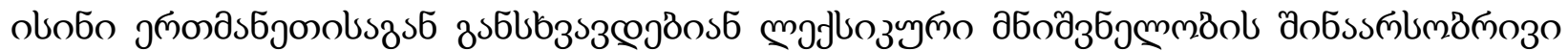

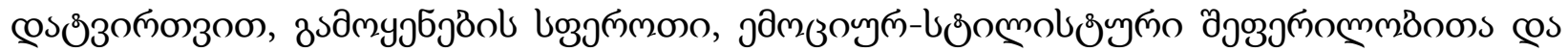

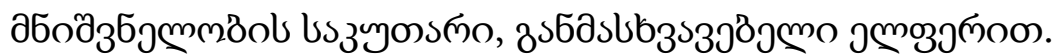

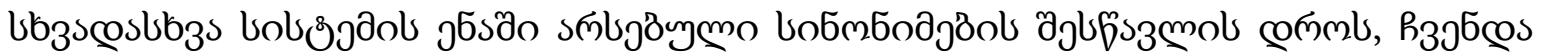

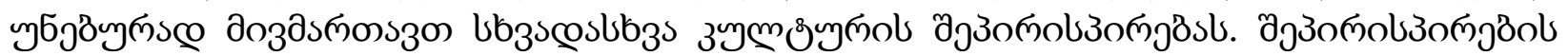

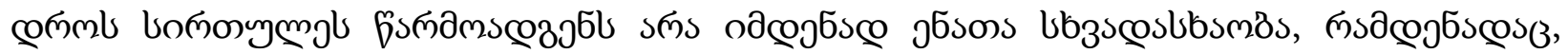

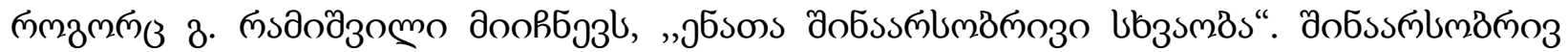

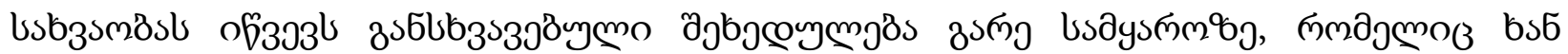

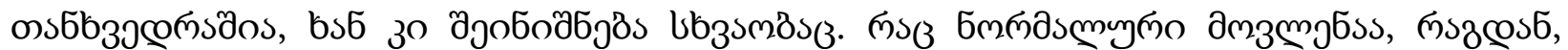

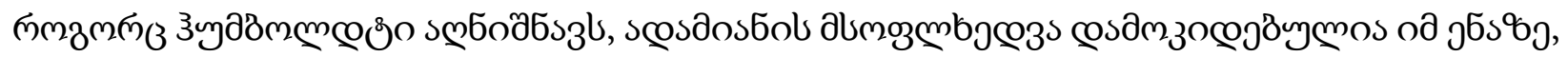

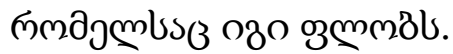

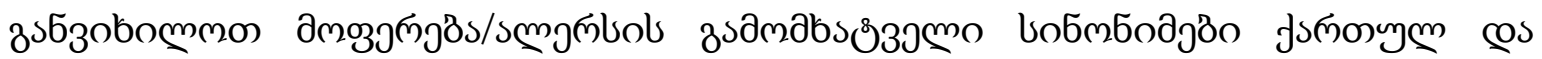
oбzmolugn jбsð̃o.

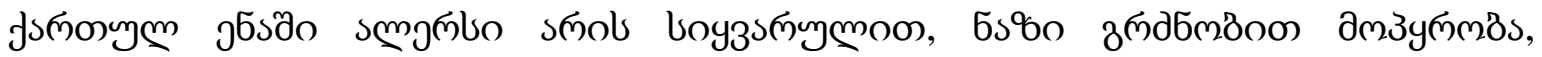

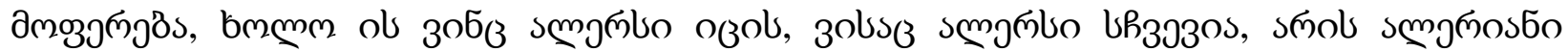

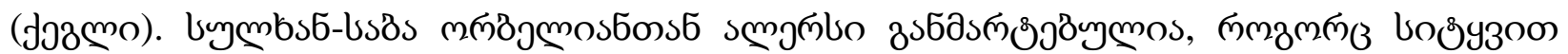

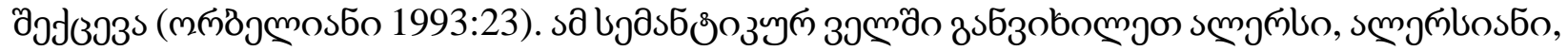

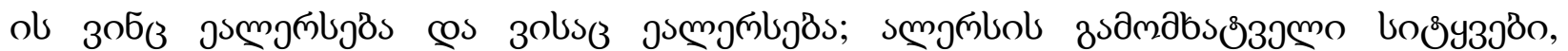

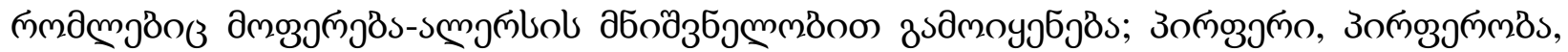

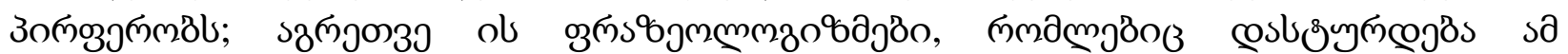

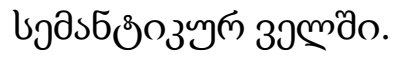

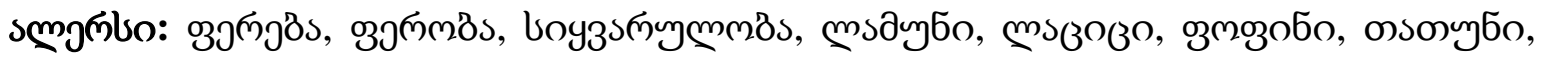

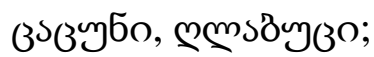

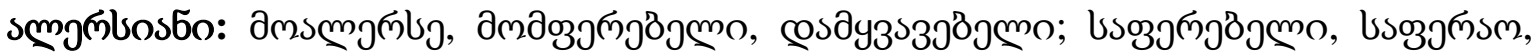
usmmलूos 3 m;

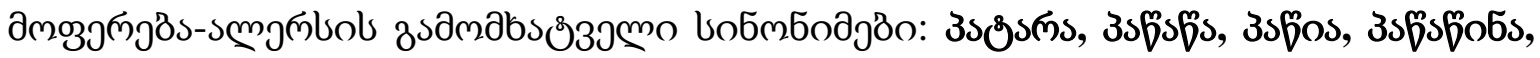

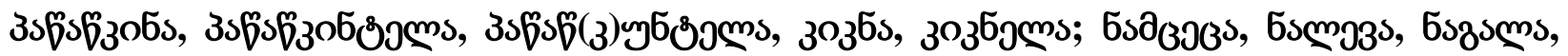

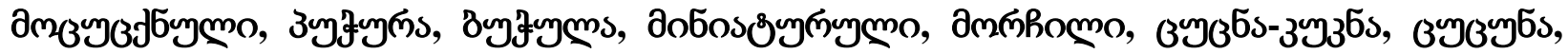

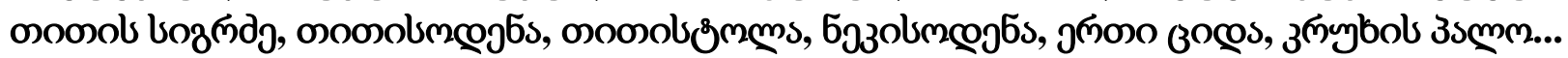

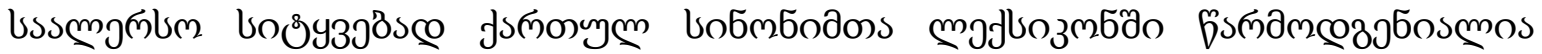

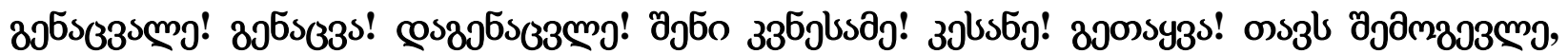

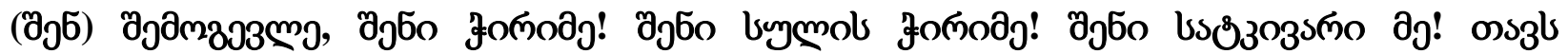

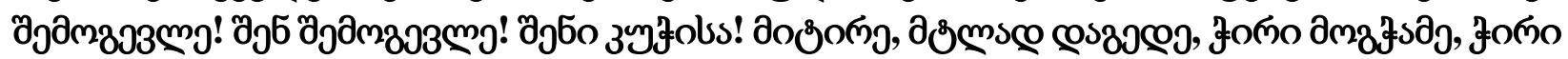

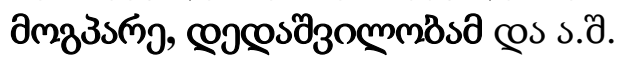

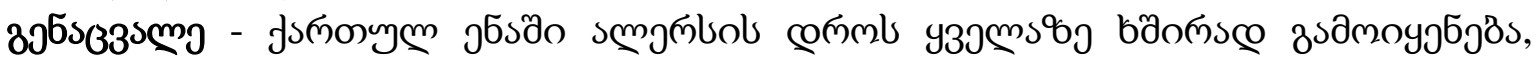

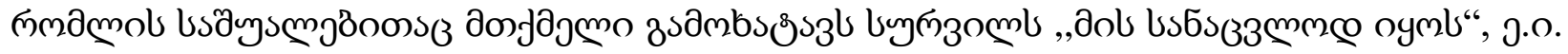




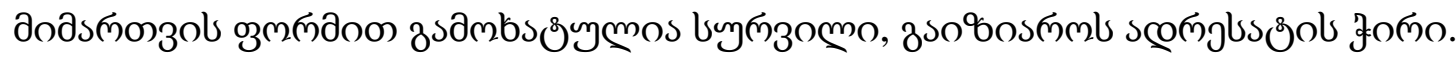

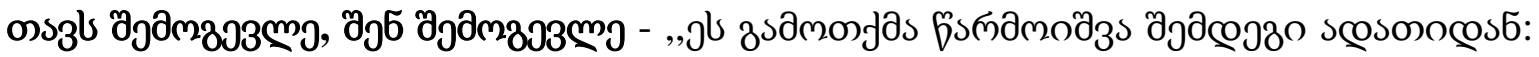

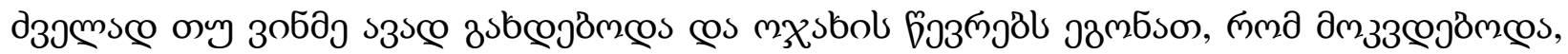

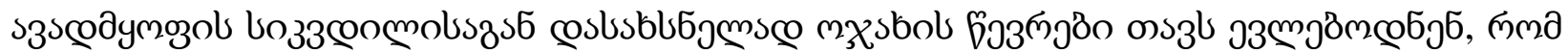

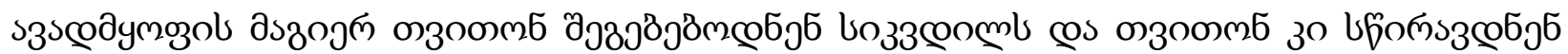

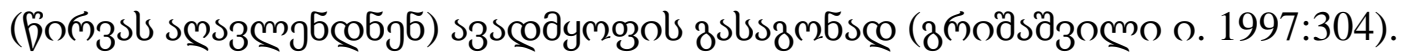

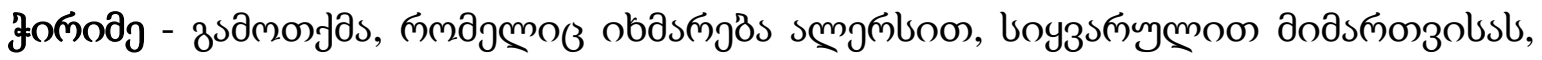

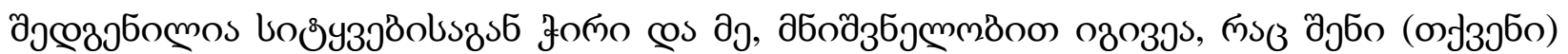

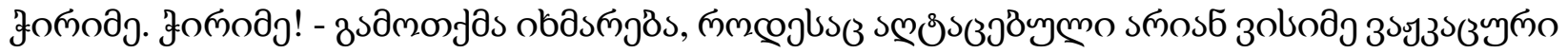

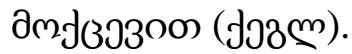

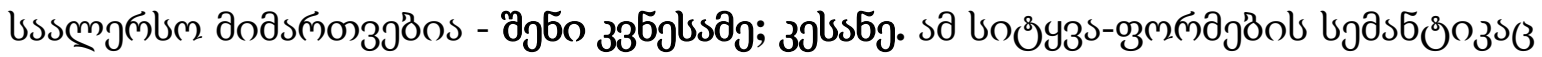

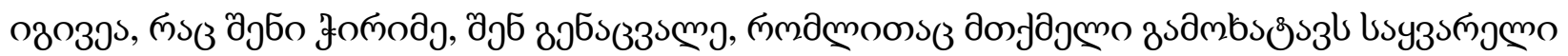

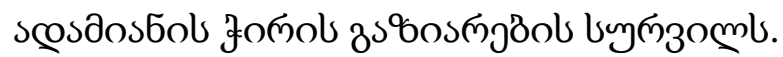

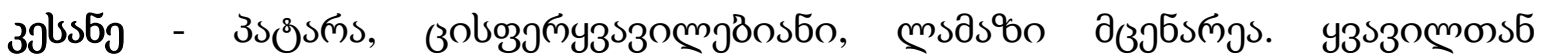

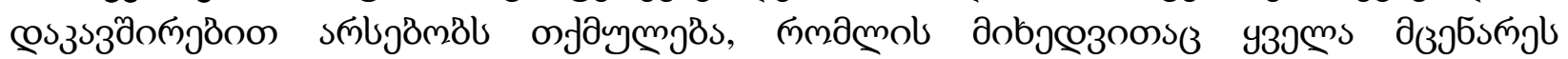

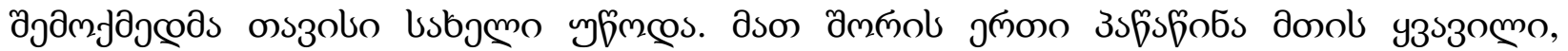

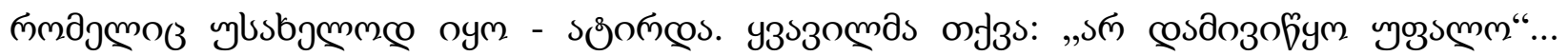

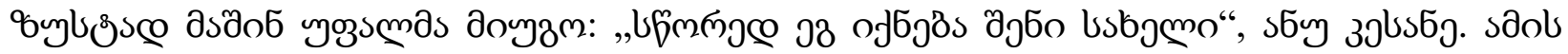

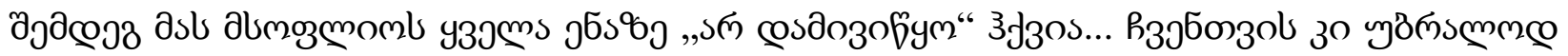

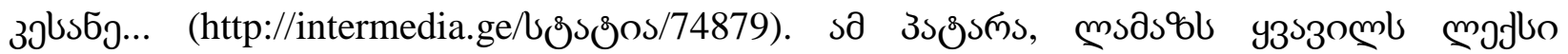

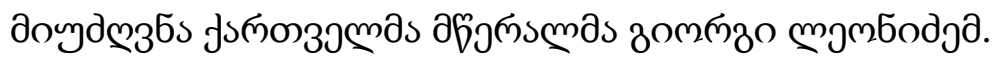

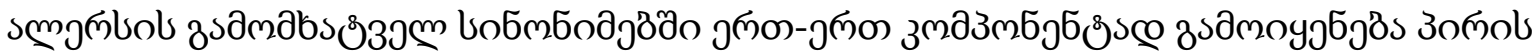

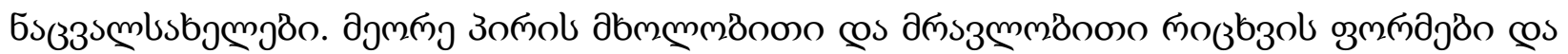

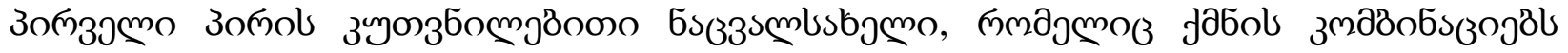

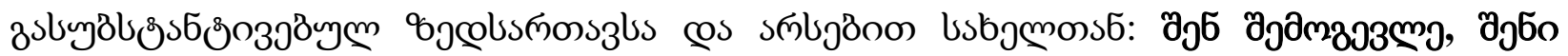

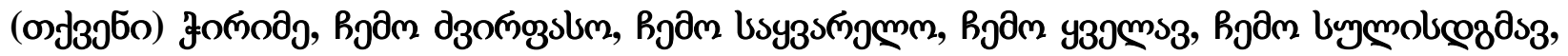

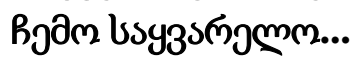

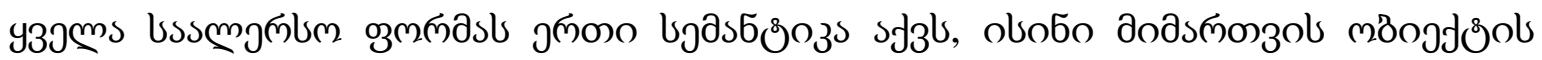

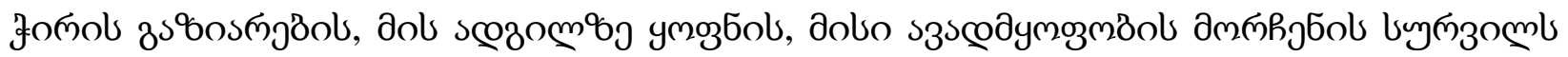
зsवmbsos 325 .

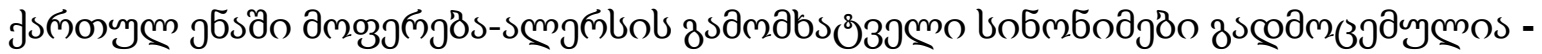

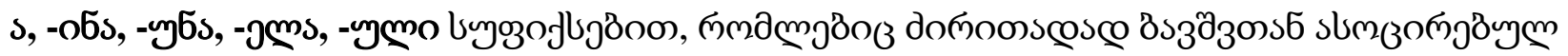

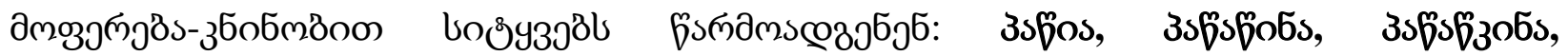

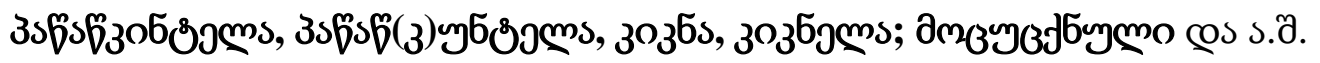

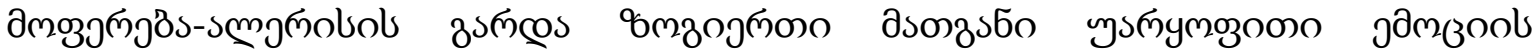

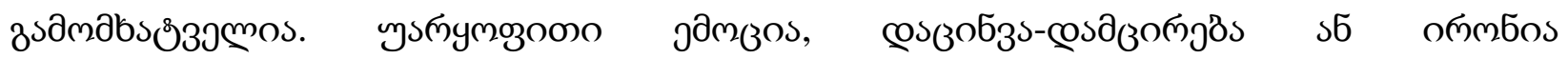

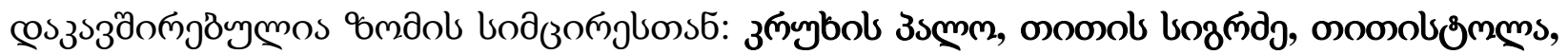

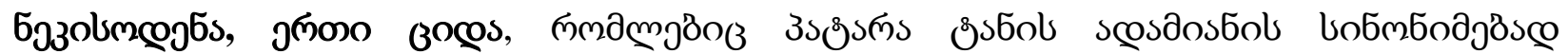

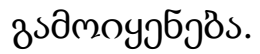

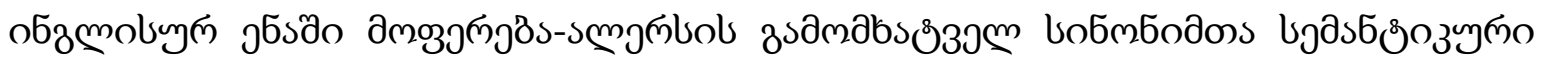

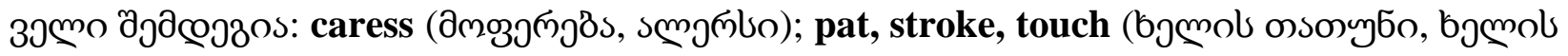




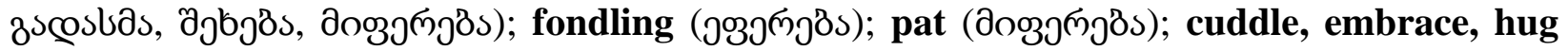

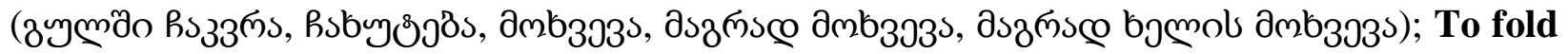

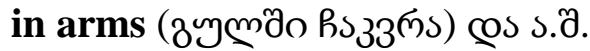

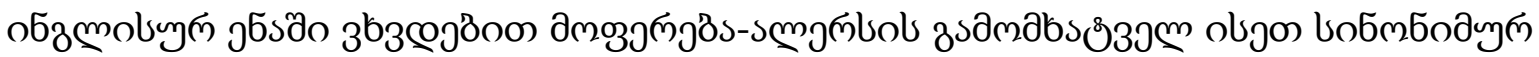

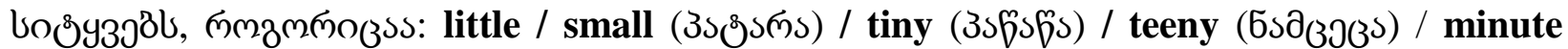

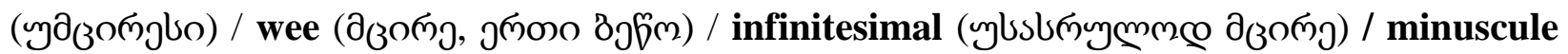

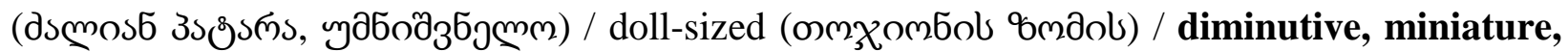

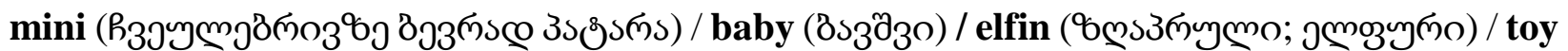

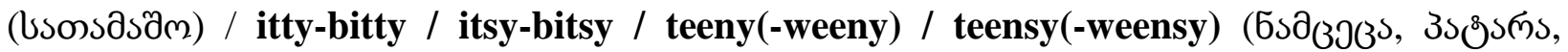

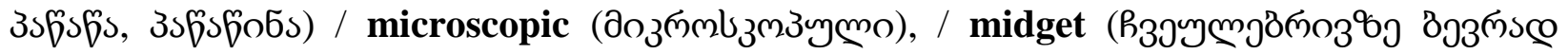

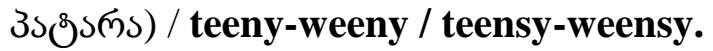

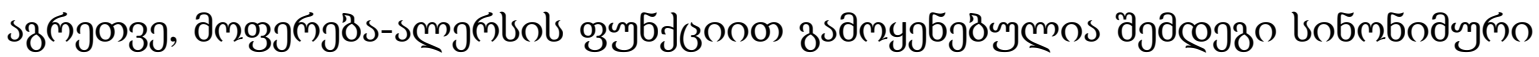

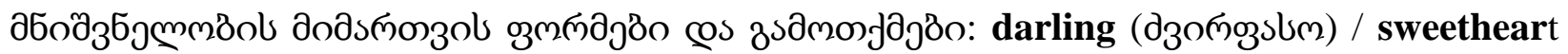

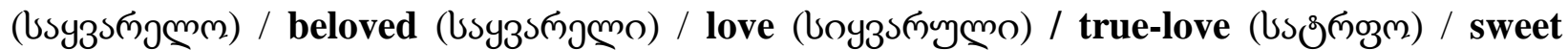
(

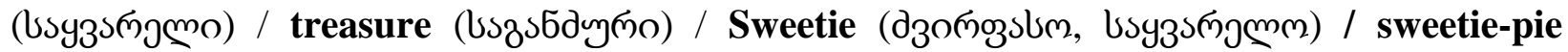

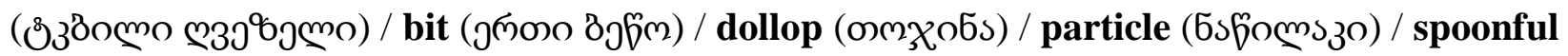

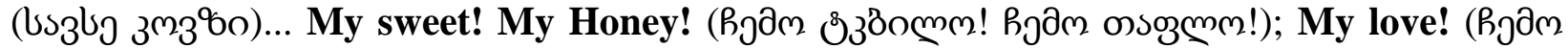

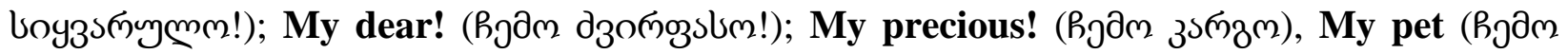

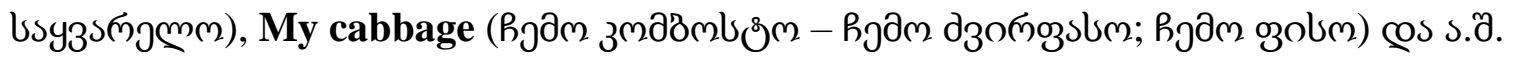

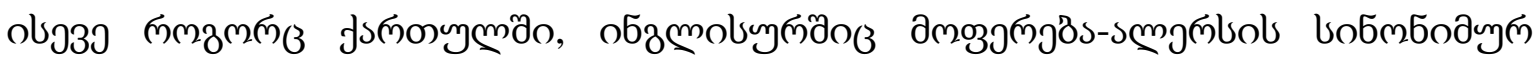

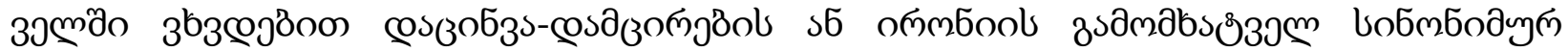

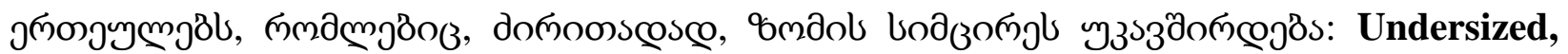

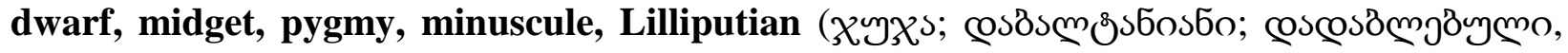

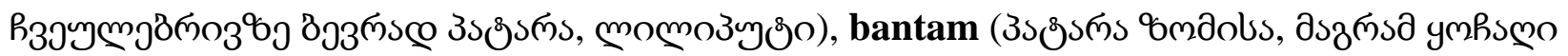

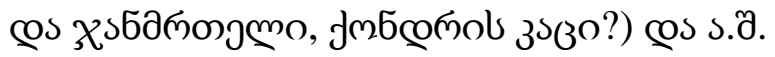

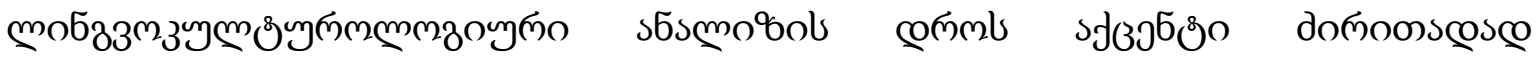

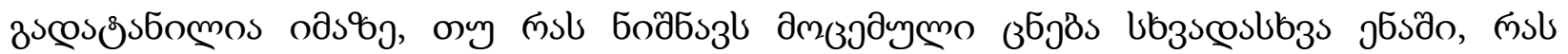

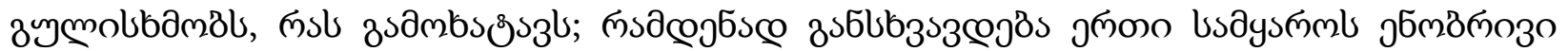

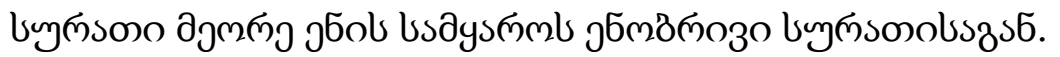

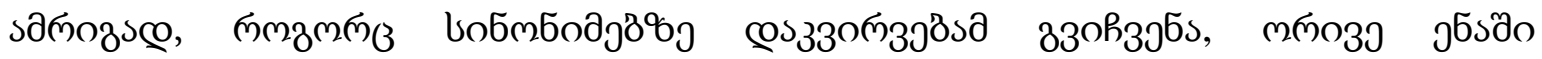

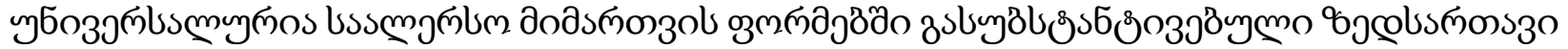

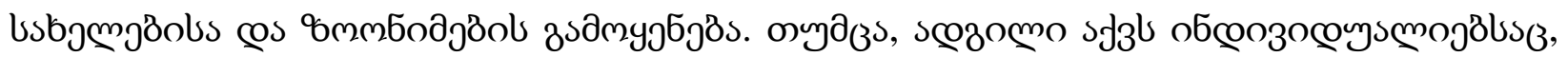

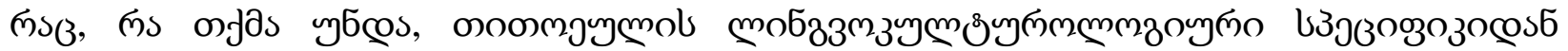

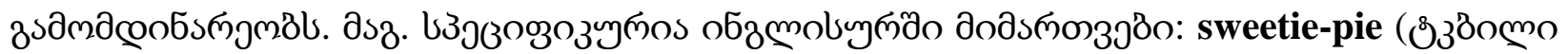
๓зэ६ бмо), bantam (

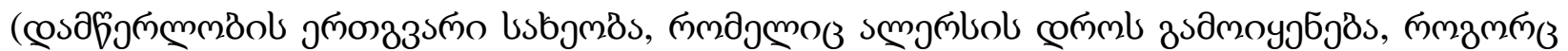

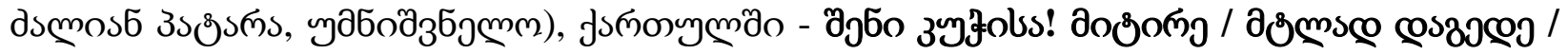

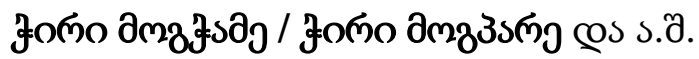




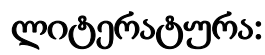

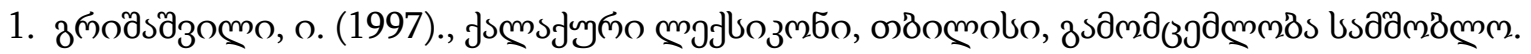

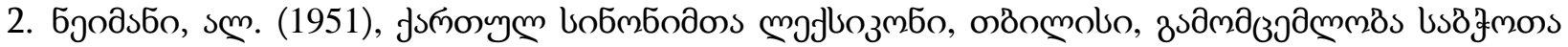

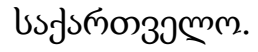

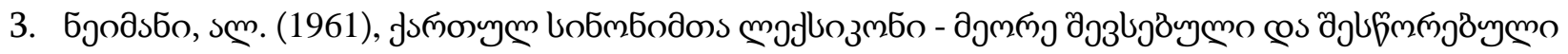

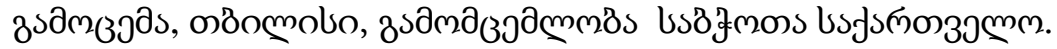

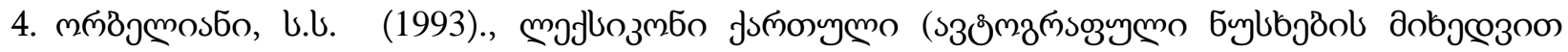

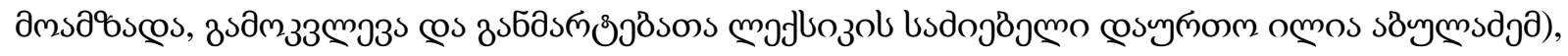

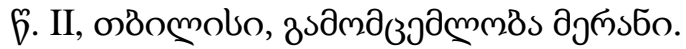

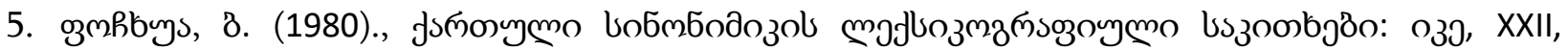

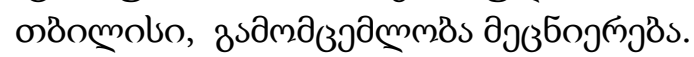

\title{
Semantic and Linguo-Cultural Analysis of Synonymic Set for Caress
}

(Based on the Georgian-English synonymic dictionaries)

\author{
Tsignadze Shorena \\ Iakob Gogebashvili Telavi State University
}

\begin{abstract}
The semantic and Lingo-cultural analysis of the synonymic et for cares is discussed in the article. Georgian and English synonymic dictionaries are used as an analytic source. Studying synonyms has quite a long history. Linguistic observation of such items in different systematic languages is very interesting. We consider the difference which exists in the Georgian and English languages as structural-semantic, as well as lingu-cultural point of views. Synonyms are the part of lexical-semantic system of a language and the main point is shown in their absolute or partial coincidence. Their study allows us to have the access to a huge reserve depictive means of a language and gives us the opportunity to show them in a methodological way, which is "very crucial for learning our mother tongue as well as a foreign language"(Jorjaneli. K. 2006:6).

While making a linguo-cultural analysis, the focus is mainly point on the question of what this notion means in different languages, what does it imply to, how is it expressed by; how does one language differ from another based on their linguistic portraits.

As the observation on synonyms has revealed, the usage of substantial adjectives and zoonyms in the caress-endearment speech is universal for these two languages. Of course, individual words aso participate, which is the result of the linguo-cultural characteristics of each
\end{abstract}


language. For example, addressing words like the following ones are very specific in English : Sweetie-pie( a sweet patty), bantam ( bantam is associated with the breed of a little domestic hen), minuscule ( this is one of script varieties. It is used while showing an affection as a sign of something very little, insignificant), and in Georgian we can find - Jj5o ł3yobs! ( Sheni chkuisa)

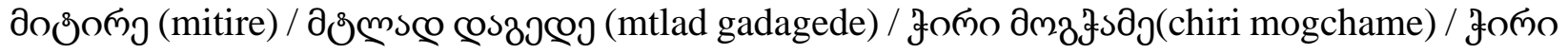
дmzउsळy (chiri mogpare) and so on.

Key words: Synonyms, semantics, linguo-culture, caress, endearment. 